\title{
Neonatal mucosal immunization with a non-living, non-genetically modified Lactococcus lactis vaccine carrier induces systemic and local Th1-type immunity and protects against lethal bacterial infection
}

\author{
K Ramirez $^{1,4}$, Y Ditamo ${ }^{1,4}$, L Rodriguez $^{1}$, WL Picking ${ }^{2}$, ML van Roosmalen $^{3}$, K Leenhouts ${ }^{3}$ and \\ MF Pasetti ${ }^{1}$
}

Safe and effective immunization of newborns and infants can significantly reduce childhood mortality, yet conventional vaccines have been largely unsuccessful in stimulating the neonatal immune system. We explored the capacity of a novel mucosal antigen delivery system consisting of non-living, non-genetically modified Lactococcus lactis particles, designated as Gram-positive enhancer matrix (GEM), to induce immune responses in the neonatal setting. Yersinia pestis LcrV, used as model protective antigen, was displayed on the GEM particles. Newborn mice immunized intranasally with GEM-LcrV developed LcrV-specific antibodies, Th1-type cell-mediated immunity, and were protected against lethal $Y$. pestis (plague) infection. The GEM particles activated and enhanced the maturation of neonatal dendritic cells (DCs) both in vivo and in vitro. These DCs showed increased capacities for secretion of proinflammatory and Th1-cell polarizing cytokines, antigen presentation and stimulation of $\mathrm{CD} 4^{+}$and $\mathrm{CD} 8^{+} \mathrm{T}$ cells. These data show that mucosal immunization with $L$. lactis GEM particles carrying vaccine antigens represents a promising approach to prevent infectious diseases early in life.

\section{INTRODUCTION}

One of the major challenges in vaccinology is the development of safe and effective vaccines that can protect newborns and infants against infectious diseases. Conventional vaccines are notoriously poorly immunogenic during the first months of life, inducing immune responses that are short-lived and heavily Th2 biased. Th1-type cell-mediated immunity is modest or absent, and the combination of these factors heightens the risk of intracellular bacterial infections. ${ }^{1-3}$ Even though routine immunization of human infants starts at 6 to 12 weeks of age, several booster doses are needed to achieve protective immunity. Mounting evidence indicates that these poor responses are not due to intrinsic deficiencies of the neonatal immune system, which has a fully constituted T-cell repertoire and is capable of responding to antigens, but mainly to the presence of immature or "inexperienced" immune cells, particularly dendritic cells (DCs), which have a limited capacity for antigen presentation and stimulation of naive T cells. ${ }^{1,3-5}$ The field of neonatal vaccinology has experienced unprecedented progress in recent years, and the literature supports the assertion that newborns are indeed capable of mounting potent adaptive immunity, including adult-like Th1-type immune responses to vaccine antigens, provided that these antigens are administered with the appropriate stimulatory signals. ${ }^{1-3,5-7}$

\footnotetext{
${ }^{1}$ Department of Pediatrics, Center for Vaccine Development, University of Maryland School of Medicine, Baltimore, Maryland, USA. ${ }^{2}$ Department of Microbiology and Molecular Genetics, Oklahoma State University, Stillwater, Oklahoma, USA. ${ }^{3}$ Mucosis BV, Groningen, The Netherlands. Correspondence: MF Pasetti (mpasetti@medicine.umaryland.edu)

${ }^{4}$ These authors contributed equally to the study. 
In this study, we examined the possibility of priming the neonatal immune system through mucosal immunization using a novel antigen delivery system consisting of non-living, nongenetically modified cell wall particles derived from Lactococcus lactis. These particles, referred to as Gram-positive enhancer matrix (GEM) particles, are produced by the heat-acid treatment of freshly grown L. lactis, a process that removes the DNA and most of the bacterial proteins, but leaves the peptidoglycan (PGN) envelope intact. ${ }^{8,9}$ Multiple antigens can be displayed on the particle surface using recombinant protein and affinity technology: recombinant fusion proteins containing a vaccine antigen and a PGN protein anchor domain that binds with high affinity to the bacterial PGN are produced in a suitable host cell, purified, and then combined with naked GEM particles. The recombinant proteins bind tightly to the GEMs, creating antigen-displaying particles. A major advantage of the GEM particles for neonatal immunization is their safety profile. L. lactis is a non-pathogenic Gram-positive, lactic acid bacterium, generally recognized as safe (GRAS) and widely used in dairy products. Probiotics have been safely given to newborns, ${ }^{10}$ young children in day care, ${ }^{11}$ and even critically ill children. ${ }^{12}$ Unlike recombinant live organisms, including attenuated pathogens, the L. lactis GEM particles do not contain DNA, i.e., there is no risk of potential reversion to a virulent form. The composition of the GEMs also contributes to their immune-stimulating properties. As spherical particles, the GEMs can be efficiently taken up by $\mathrm{M}$ cells in the epithelium above the mucosal lymphoid follicles, and the transported antigens can be delivered directly to the underlying DCs in mucosal inductive sites. Furthermore, the PGN envelope is a potent stimulator of innate immunity. ${ }^{13}$

We used Yersinia pestis LcrV as a model vaccine antigen to demonstrate the feasibility of successful early life immunization using the GEM platform technology. The immunogenicity and protective efficacy of $L$. lactis GEM particles displaying $Y$. pestis LcrV was investigated in a neonatal mouse model. We showed, for the first time, that intranasal immunization of newborn mice with GEM-LcrV elicits a potent mucosal and systemic immunity that protects against lethal systemic plague infection. We also demonstrated that the L. lactis GEM particles enhance the maturation of neonatal CD11 $\mathrm{c}^{+} \mathrm{DC}$, and that these cells have increased capacity for secretion of proinflammatory and Th1-type-promoting cytokines and can stimulate antigen-specific interferon (IFN)- $\gamma$-secreting CD $4^{+} \mathrm{T}$ cells. Furthermore, we showed that the GEM particles were taken up by DCs from human newborns and that these cells also acquired a mature phenotype such that they were able to stimulate human $\mathrm{T}$ cells. Together, these results indicate that mucosally delivered antigendisplaying $L$. lactis GEM particles represent a highly promising vaccine approach for immunization early in life.

\section{RESULTS}

\section{L. lactis GEM particles induced maturation of neonatal and adult mouse DCs}

To ascertain whether the L. lactis GEM particles could provide strong immunological signals to stimulate the neonatal immune system, we first examined the ability of the GEM particles to activate and enhance the functional capacity of neonatal DCs. The expression of activation and maturation cell-surface markers CD80, CD86, CD40, and major histocompatibility complex (MHC)-class II (I-Ad) was measured on bone marrow (BM)-derived CD11 $\mathrm{c}^{+}$cells from newborn (7-day-old) mice stimulated with L. lactis GEM particles or mock-stimulated cells (Figure 1a). To determine the strength of the activation of GEM-stimulated neonatal DCs in comparison with that of adult DCs, BM-derived CD11c ${ }^{+}$cells from 6- to 8-week-old mice were included in all experiments. All markers were upregulated in neonatal and adult DCs after GEM stimulation, compared with the mock-treated DCs (Figure 1a). Neonatal GEMexposed DCs exhibited a noticeable increase in the expression of CD86, while both CD86 and MHC-II were the markers most abundantly expressed on adult GEM-stimulated DCs. A summary of the increases in the expression of cell-surface markers in both neonatal and adult DC exposed to the L. lactis GEMs or to Escherichia coli lipopolysaccharide (LPS) (used as positive control) is shown in Table 1. It is noted that the upregulation of MHC-II and costimulatory molecules in both neonatal and adult GEM-stimulated DCs was remarkably similar to that induced by LPS under the same experimental conditions.

\section{DC stimulated with $L$. lactis GEMs secreted proinflammatory cytokines}

To assess the functional capacity of the GEM-activated DCs, we examined their ability to secrete proinflammatory, Th1-promoting and regulatory cytokines, as these are all critical signals required for the development of an effective adaptive immune response. The levels of interleukin (IL)-12p70, tumor necrosis factor (TNF)- $\alpha$, IL-10, IL-6, IFN- $\gamma$, and MCP- 1 were measured in culture supernatants from neonatal and adult BM-derived CD $11 \mathrm{c}^{+}$cells treated with L. lactis GEMs or LPS, or from mock-treated cells. For all of the cytokines tested, both neonatal and adult GEM-stimulated DCs had an increased capacity for cytokine production as compared with mock-treated cells (Figure 1b). The levels of cytokines produced by both adult and neonatal mouse DCs stimulated with the L. lactis GEM particles were comparable, suggesting that neonatal GEM-activated DC can reach a degree of functional activity similar to that of adult mature DCs. Interestingly, the fold-increases for TNF- $\alpha$ and IL- 6 were much higher in DC from the newborns regardless of whether they were stimulated with the GEMs or LPS (Table 1).

\section{GEM-exposed mature DCs had reduced capacity for antigen uptake}

During the process of maturation, DCs relent their endocytic and phagocytic activity as they become more efficient antigen-presenting cells. ${ }^{14}$ Accordingly, we examined the capacity of GEM-stimulated DCs to incorporate fluorescein isothiocyanate (FITC)-dextran as a measure of antigen uptake. Both neonatal and adult CD11 $\mathrm{c}^{+} \mathrm{DCs}$ exposed to the GEM particles had a markedly lower capacity to incorporate FITC-dextran (Figure 1c). Compared with mock-stimulated DCs, the adult and neonatal DCs showed 85 and $90 \%$ reduction 

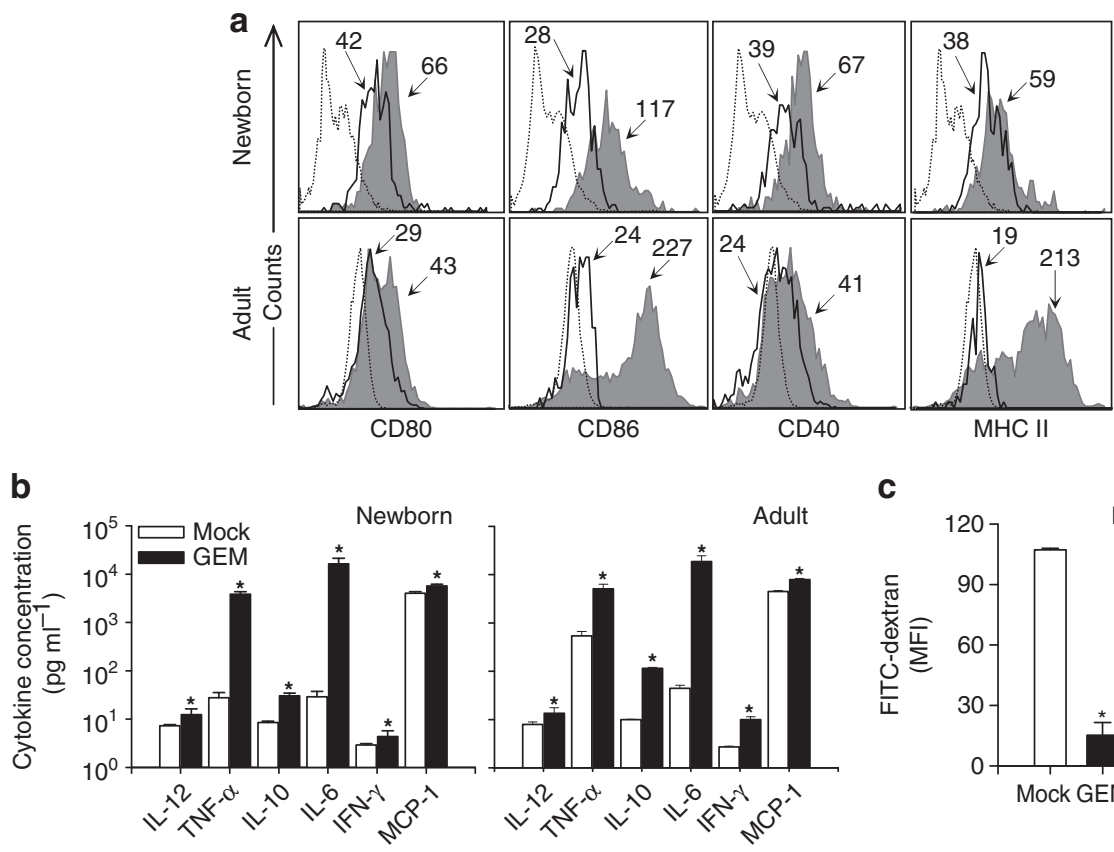

c
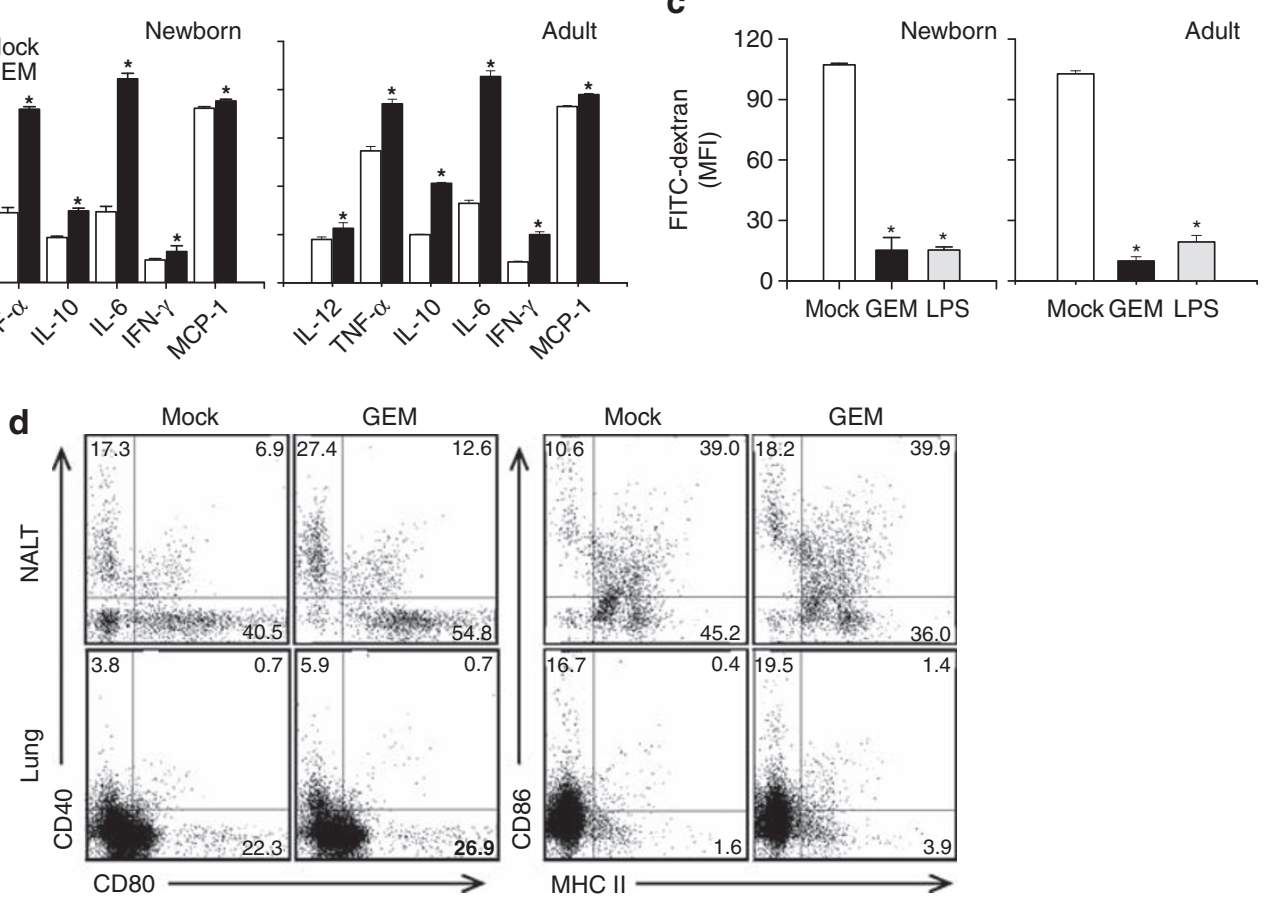

Figure 1 Lactococcus lactis GEM particles enhanced maturation and cytokine production of neonatal and adult mouse DC. (a) Expression of activation and maturation cell-surface markers CD80, CD86, CD40, and MHC class II on neonatal and adult mouse BM-derived CD11 ${ }^{+}$DC treated with GEM particles (shaded area) or mock-treated (solid line); the dashed line indicates isotype control staining. The mean fluorescence intensity (MFI) on CD11 $\mathrm{c}^{+}$gated cells is indicated. (b) Cytokines produced by neonatal and adult mouse BM-derived CD11 ${ }^{+}$DC stimulated with GEM particles or mock-stimulated, measured in culture supernatants. Data are representative of three independent experiments. (c) FITC-dextran uptake by neonatal and adult $\mathrm{CD} 11 \mathrm{c}^{+} \mathrm{DC}$ exposed to GEM or E. coli LPS (positive control), or mock-treated DC, measured by flow cytometry; data represent MFI \pm S.d. from three independent experiments. Significant differences $(P<0.001)$ compared with mock-stimulated cells are denoted by an asterisk $\left(^{*}\right)$. (d) Activation and maturation of neonatal DC exposed to GEM particles in vivo. Mice were immunized with GEMs or PBS (mock) on day 7 after birth and NALT and lungs were harvested on day 15. The number in each quadrant indicates the percentage of CD11 ${ }^{+}$DC expressing activation and maturation cell-surface markers CD40, CD80, CD86, and MHC class II. BM, bone marrow; DC, dendritic cell; GEM, Gram-positive enhancer matrix; LPS, E. coli lipopolysaccharide; NALT, nasal-associated lymphoid tissue.

in FITC-dextran incorporation, respectively, further attesting to their mature phenotype.

\section{In vivo activation of mucosal neonatal mouse DCs by the GEM particles}

To confirm that the in vitro process of neonatal DC maturation mediated by the GEM particles also occurs in vivo, we examined the phenotype of CD11 $\mathrm{c}^{+} \mathrm{DC}$ in the nasal-associated lymphoid tissue (NALT) and the lungs of 15-day-old mice that had been immunized with the L. lactis GEM particles at 7 days of age. Figure 1d shows the expression of CD40, CD80, CD86, and MHC-class II on CD11 ${ }^{+}$cells. As observed in vitro, DCs from newborn mice exposed in vivo to the GEM particles also exhibited a more activated and mature phenotype as compared with the naive (mock-exposed) controls. A remarkable increase in the number of $\mathrm{CD} 11 \mathrm{c}^{+}$cells expressing both $\mathrm{CD} 40$ and $\mathrm{CD} 80$ was seen in the NALT. A more pronounced level of activation was observed in this tissue as compared with the lungs, consistent with the proximity of the nasal tissue to the site of inoculation.

\section{GEM particles activated neonatal and adult DCs through TLR2} We hypothesized that the recognition of GEM particles by DCs and their subsequent activation was mediated through toll-like receptor (TLR) signaling. Thus, we investigated the capacity of 
Table 1 Summary of fold-increases in the expression of surface markers and in cytokine production by human and mouse CD11c + DC stimulated with L. lactis GEM, E. coli LPS (mDC), or TNF- $\alpha$ (hDC) as compared with mock-treated DC

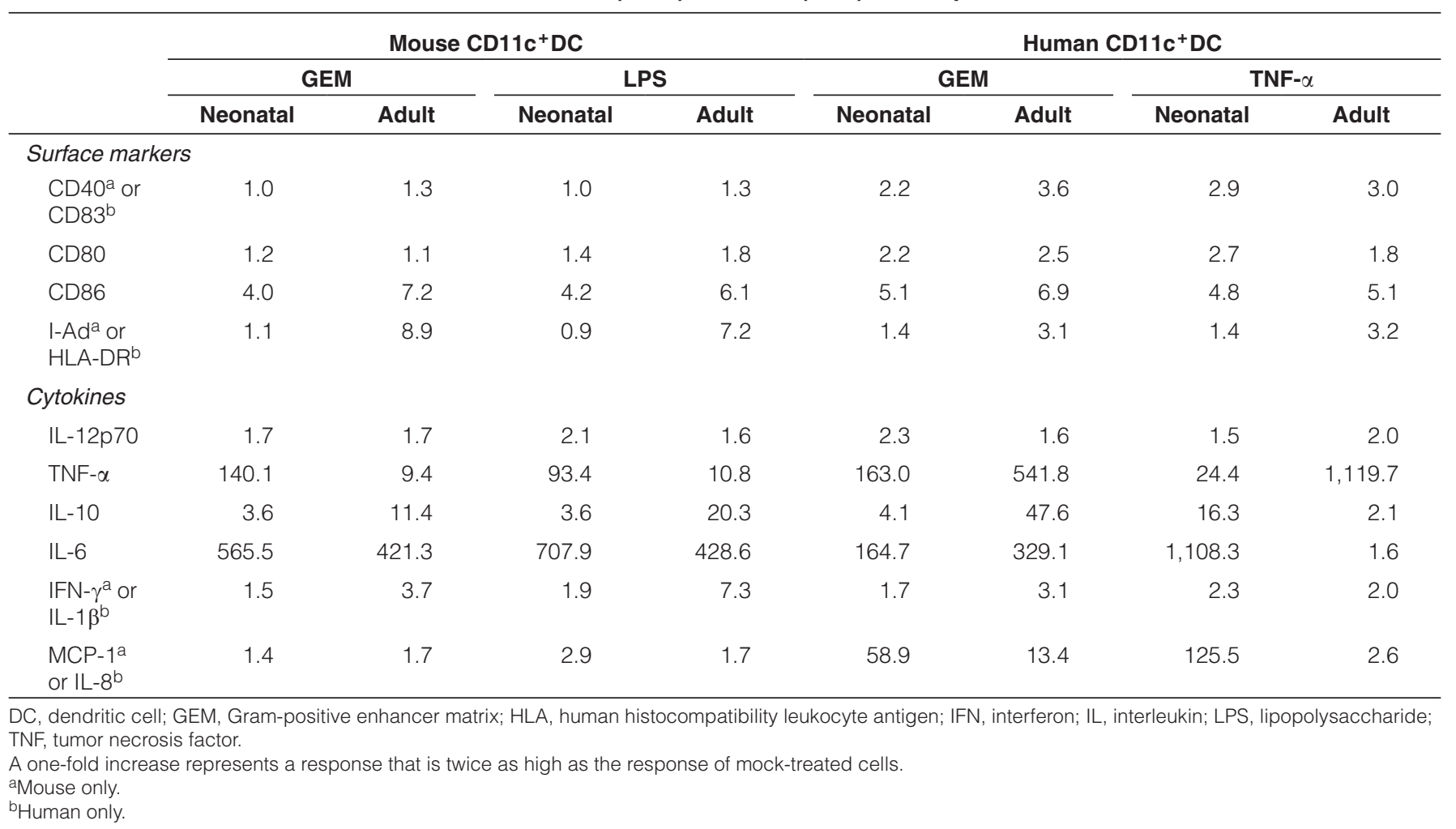

the GEMs to bind a panel of human and mouse TLRs expressed in HEK293 cells. Nuclear factor- $\kappa B$ activity was measured and used as a readout for activation. The GEM particles interacted with human TLR2 but not with any of the other TLRs tested (human TLR3, TLR4, TLR5, TLR7, TLR8 and TLR9, and mouse TLR7 and TLR8; Figure 2a). The capacity of the GEM particles to signal through TLR2 expressed on neonatal and adult mouse DCs was confirmed by antibody inhibition. In this assay, the production IL-6, detected in culture supernatants, was used as a measure of TLR2 activation. CD11 $\mathrm{c}^{+}$DCs preincubated with increasing amounts of TLR2-specific antibodies and subsequently stimulated with the GEM particles produced significantly lower levels of IL-6 than untreated DCs (or DC incubated with isotype control). The level of IL-6 produced decreased in a dose-dependent manner, further confirming the specificity of the antibody blockage and the TLR2 involvement (Figure 2b).

\section{Enhancement of neonatal antigen presentation and stimulation of antigen-specific $T$ cells}

We used the Y. pestis LcrV as a model antigen to test the ability of the L. lactis GEMs to prime an immune response in the neonatal setting. LcrV-based vaccines have been shown to induce complete protection against $Y$. pestis challenge in animal models (reviewed by Smiley ${ }^{15}$ ). However, none of these vaccines has been tested for tolerability and efficacy in young hosts. We therefore undertook the study of the GEM-LcrV system as a plague vaccine candidate for neonatal and infant immunization. LcrV-PGN binding domain fusions were produced in a recombinant $L$. lactis and attached externally to the GEM particles, as described in Figure 3.
We first examined in vitro the capacity of neonatal DCs exposed to LcrV-carrying GEM particles to process the vaccine antigen and present it to $\mathrm{T}$ cells. To this end, neonatal CD11 $\mathrm{c}^{+}$ DCs pretreated with GEM-LcrV or LcrV, or mock-treated DCs, were incubated with $\mathrm{LcrV}$-specific $\mathrm{CD} 4^{+}$and $\mathrm{CD} 8^{+} \mathrm{T}$ cells. Lymphocyte proliferation and the frequency of IFN- $\gamma$-secreting $\mathrm{CD} 4^{+} \mathrm{T}$ cells were measured as readouts of T-cell activation (Figure $4 \mathbf{a}$ and $\mathbf{b}$ ). Both $\mathrm{CD} 4^{+}$and $\mathrm{CD} 8^{+} \mathrm{T}$ lymphocytes showed remarkable proliferative responses when stimulated with neonatal DCs previously exposed to GEM-LcrV. These responses largely surpassed those of $\mathrm{T}$ cells stimulated with LcrV- or mock-treated DCs. The frequency of LcrV-specific IFN- $\gamma$-secreting CD4 ${ }^{+} \mathrm{T}$ cells also increased sharply and in a dose-dependent manner when $\mathrm{T}$ cells were stimulated with DCs treated with GEM-LcrV, as opposed to stimulation with LcrV- or mock-treated DCs, both of which failed to induce a significant IFN- $\gamma$ response (Figure $\mathbf{4 b}$ ).

\section{Immunization with GEM-LcrV elicited potent mucosal and systemic immunity early in life}

We next evaluated the immunogenicity and protective efficacy of the GEM-LcrV particles in vivo. Newborn mice were immunized intranasally (i.n.) on days 7, 15, and 21 after birth, with GEM-LcrV, recombinant LcrV, or GEM alone (Figure 5a). A group immunized intramuscularly (i.m.) with recombinant LcrV adsorbed to alum was included as control. The GEM-LcrV particles elicited high levels of LcrV-specific serum immunoglobulin $\mathrm{G}$ (IgG) antibodies that were first detected on day 21 after birth; $80 \%$ of the animals seroconverted at this 


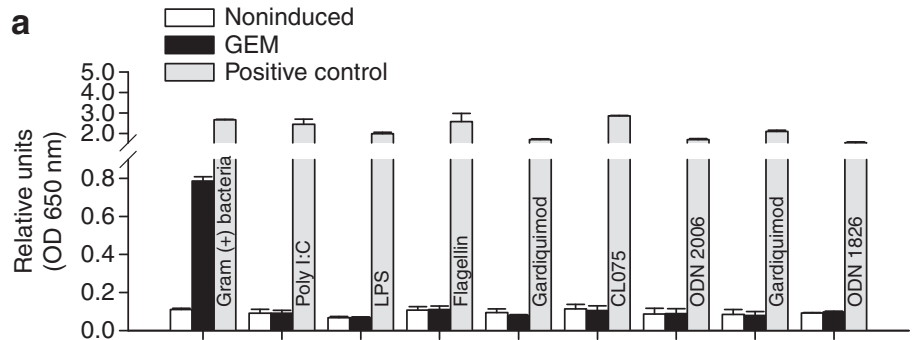

hTLR2 hTLR3 hTLR4 hTLR5 hTLR7 hTLR8 hTLR9 mTLR7 mTLR8 b

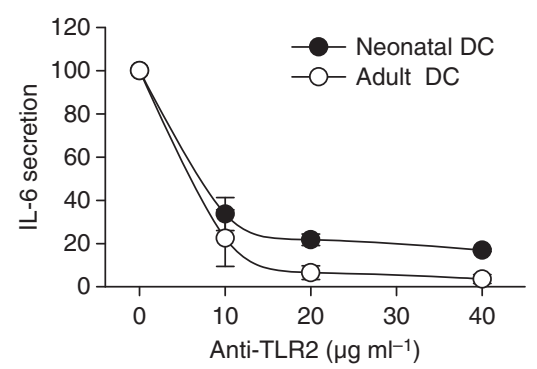

Figure 2 Lactococcus lactis GEM particles activate neonatal and adult mouse DC and evoke IL-6 production through TLR2 activation. (a) GEM interaction with human $(\mathrm{h})$ and mouse $(\mathrm{m})$ TLRs individually expressed on HEK93 cells, as measured by nuclear factor- $\mathrm{KB}$ activation using a phosphatase alkaline reporter gene. Data represent relative units for OD $650 \mathrm{~nm} \pm$ s.d. from duplicate screening experiments. (b) Inhibition of IL-6 production by GEM-stimulated neonatal and adult mouse CD11 ${ }^{+}$DC in the presence of TLR2-blocking monoclonal antibody. TLR2 inhibition curve shows relative cytokine levels (expressed as a percentage of the max. IL-6 production by GEM-stimulated DC in the absence of TLR blocking) measured by ELISA in culture supernatants; data are representative of two independent experiments. DC, dendritic cell; ELISA, enzyme-linked immunosorbent assay; GEM, Gram-positive enhancer matrix; IL, interleukin; TLR, toll-like receptor.

\section{a}

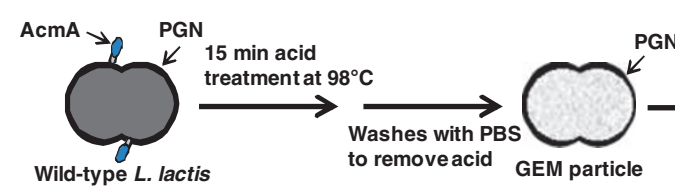

b

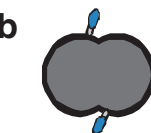

Wild-type L. lactis

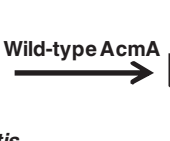

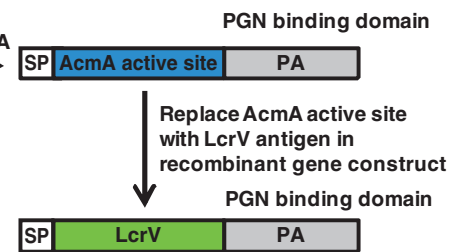

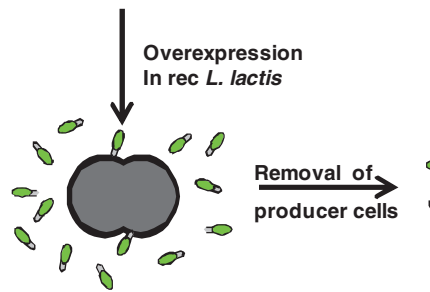

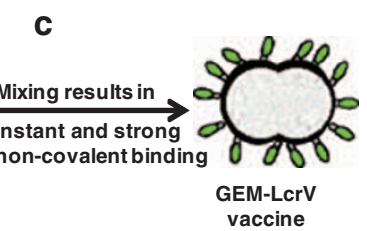

vaccine d

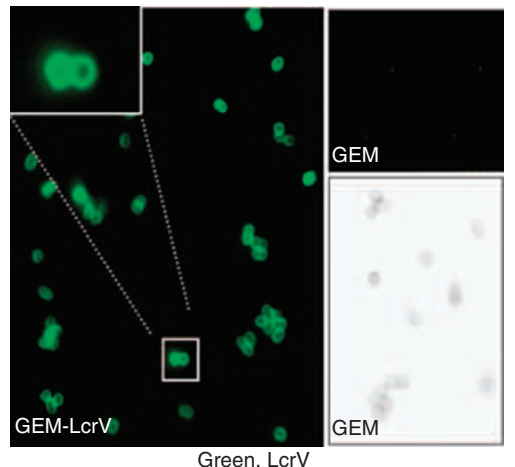

$0 \infty 8$

$\infty 88 \infty$

$\rho_{0}^{\infty} \infty$

Figure 3 Schematic representation illustrating the preparation of L. lactis GEM particles displaying Y. pestis LcrV. (a) GEM particles are obtained by heat-acid treatment of $L$. lactis followed by extensive washing. The resulting bacterial "shell" (GEM) particles have an appearance and dimensions similar to those of the living organism. L. lactis contains an extracellular cell-wall degrading enzyme (hydrolase AcmA), which in its natural form allows separation of daughter cells by cleaving the PGN wall during bacterial division. This enzyme is naturally exported and attaches (from the outside) to the bacterial cell wall though a C-terminal binding domain that has high affinity for the L. lactis PGN. The use of this PGN binding domain allows for a strong non-covalent binding of the fusion proteins to GEM particles. (b) The AcmA locus, containing the enzyme active site next to the PGN binding domain and preceded by a signal peptide (SP), was cloned into an expression plasmid (pPA3). LcrV was inserted in lieu of the AcmA active site on pPA3 so that the LcrV sequence is in tandem with the PGN binding domain. The resulting plasmid, pPA281, was introduced into live L. lactis, which then produced the LcrV-PGN binding domain fusion as a recombinant protein and released it into the culture medium. The producer cells were removed by centrifugation, and the antigen was concentrated and used for particle coating (no additional purification is needed). (c) GEM particles and LcrV-PGN binding domain fusion protein were mixed, resulting in immediate non-covalent binding of LcrV to the PGN layer. (d) Fluorescence microscopy showing GEM particles displaying LcrV using an LcrV-specific monoclonal antibody; no staining was observed with GEM alone (shown on a light microscopy image). GEM, Gram-positive enhancer matrix; PA, PGN protein anchor domain; PGN, peptidoglycan.

time. The IgG levels increased on day 28 after birth, 1 week after the last immunization, and remained increased up to day 77, the last time point tested (Figure 5b). In contrast, no responses were detected in mice immunized i.n. with GEMs or LcrV alone. The control group immunized i.m. with LcrV-alum developed IgG responses, but they peaked on day 56 after birth (5 weeks after the last immunization) and started to decrease soon after (IgG mean titers on days 56 and 77 were 137,708.1 and $62,262.9 \mathrm{EU} \mathrm{ml}^{-1}$, respectively). Both IgG1 and IgG2a were produced in mice that received GEM-LcrV or LcrV-alum 

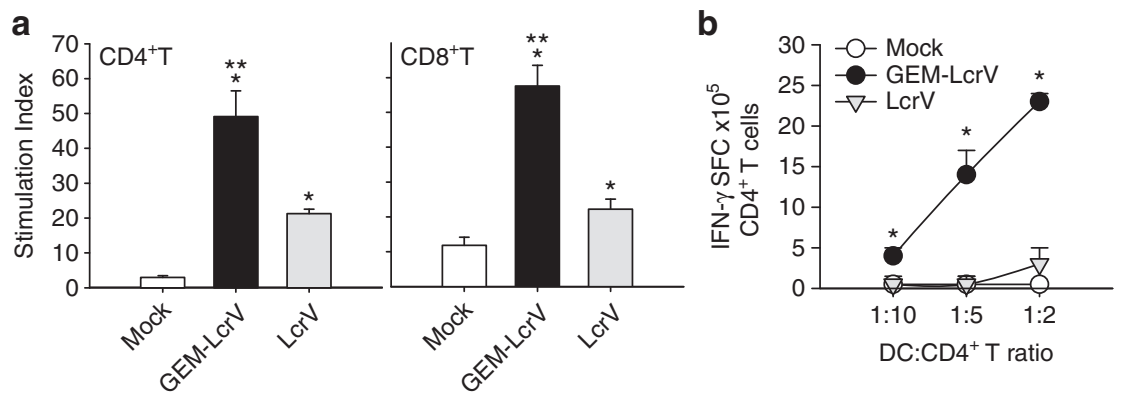

Figure 4 Lactococcus lactis GEM-LcrV enhanced antigen presentation by neonatal mouse DC and stimulation of LcrV-specific T cells. (a) Stimulation of LcrV-specific CD4 ${ }^{+}$and $C D 8^{+} \mathrm{T}$ cells by $\mathrm{CD} 11 \mathrm{c}^{+}$neonatal DC previously exposed to GEM-LcrV or LcrV, or mock-treated DC (DC:T cell ratio 1:2). Cell proliferation was measured by $\left[{ }^{3} \mathrm{H}\right]$ thymidine incorporation; data represent mean stimulation index \pm s.e.m. from replicate cultures. (b) Frequency of IFN- $\gamma$-secreting CD4 ${ }^{+}$T cells stimulated with GEM-LcrV-, LcrV- or mock-treated CD11c ${ }^{+}$neonatal DC (at increasing DC:T cell ratios) measured by ELISPOT. Data show mean IFN- $\gamma$ spot forming cells (SFC) \pm s.e.m. from replicate cultures. Significant differences compared with the mock-treated $\left({ }^{\star} P<0.002\right)$ and LcrV-treated DC $\left({ }^{* \star} P<0.01\right)$ are indicated. DC, dendritic cell; ELISPOT, enzyme linked immunospot assay; GEM, Gram-positive enhancer matrix; IFN, interferon.

(Figure 5c). However, the proportion of IgG2a was larger in the GEM-LcrV group, resulting in a higher IgG2a/IgG1 ratio, as compared with the group that received LcrV-alum (Figure 5c).

Increased levels of LcrV-specific IgM were observed in mice immunized with GEM-LcrV on day 21 after birth, which exceeded those of all the other groups (Figure 5d). Immunization with GEM-LcrV also produced high LcrVspecific serum IgA responses that were first seen on day 21 ; IgA levels peaked on day 28 and remained steady thereafter (Figure 5e). The similarity between the kinetics of IgG and $\operatorname{IgA}$ production is noteworthy, with both curves showing faster responses following immunization with GEM-LcrV over LcrV-alum (Figure $\mathbf{5 b}$ and $\mathbf{e}$ ).

We also examined the presence of LcrV-specific antibodysecreting cells (ASCs) in the spleen and BM, which are believed to represent reservoirs of vaccine-induced plasma cells that support the production and maintenance of circulating antibodies. Significant IgG LcrV ASC responses were found in mice immunized with GEM-LcrV but not in mice immunized with $\mathrm{LcrV}$ or GEMs alone. The positive control group immunized with LcrV-alum also lacked IgG LcrV ASC responses (Figure 5f).

The induction of mucosal immunity was assessed by measuring the frequency of IgA and IgG ASCs in the NALT (Figure 5g). Mice immunized i.n. with GEM-LcrV exhibited high numbers of IgA and IgG ASCs on day 21 after birth, coinciding with the appearance of serum antibodies. IgG ASCs were also found in the lungs 21 days after birth, albeit at a much lower frequency (data not shown). No mucosal ASC responses were ever detected in any of the other groups.

We also investigated the induction of cell-mediated immunity by measuring the proliferative responses of $\mathrm{LcrV}$-specific $\mathrm{T}$ cells in the spleen at different time points. Newborn mice immunized with GEM-LcrV showed a significant T-cell response on day 15 after birth, and this response further increased on day 56 ; in contrast, responses were negligible in mice immunized i.n. with GEMs or LcrV alone (Figure 5h). Mice that received LcrV-alum had a positive response on day 56 , albeit at a significantly lower level compared with those of mice that received GEM-LcrV.
Finally, we examined the cytokine profile induced in response to GEM-LcrV immunization, in the NALT and lungs as critical mucosal lymphoid tissues. These measurements were performed 21 days after birth, $\sim 1$ week after the second immunization. Th1, Th2, proinflammatory, and T-regulatory cytokines were measured in the culture supernatants of cells stimulated in vitro with LcrV- or mock-stimulated cells. We included in this experiment only the most relevant groups: newborn mice immunized i.n. with GEM-LcrV or GEMs alone, and mock-immunized (naive controls). Increased levels of IL-2 and IL-12 (p40 and p70) were observed in the NALT of newborns immunized with GEMLcrV but not in those that received GEMs alone (Figure 5i). IL- 6 , TNF- $\alpha$, and IL-10 were also increased, but this increase occurred in both groups (GEMs and GEM-LcrV), suggesting a broad and nonspecific stimulation of neonatal immune cells induced by the GEMs alone. This nonspecific stimulation was not seen in naive mice. Lung cells from newborn mice immunized with GEM-LcrV produced remarkably high levels of IFN $-\gamma$ and showed increased production of IL- 2 and IL- 6 upon in vitro antigen stimulation, none of which was seen in mice immunized with GEMs alone or in naive controls. Marginal levels of IL-5 were found in the lungs of GEM-LcrV-immunized mice, whereas IL- 4 was negligible in both tissues; IL- 5 and IL-4 were undetectable in mice that received GEMs alone or in naive controls.

\section{GEM-LcrV protected newborn mice against systemic lethal plague}

Mice immunized as newborns with GEM-LcrV, LcrV, and GEMs alone, along with naive controls, were challenged intravenously with $50 \%$ lethal doses of $Y$. pestis EV76, 8 weeks after the last immunization (Figure 6). The attack rate in naive mice was $100 \%$. These animals showed significant weight loss and severe disease, and all died by day 6 after challenge. In contrast, all mice immunized with GEM-LcrV were protected. Some of these animals showed minimal weight variations and mild signs of disease during the first week after challenge, but completely recovered thereafter. Only $20 \%$ of the animals immunized i.n. with LcrV or GEMs alone survived after challenge; mice in 


\begin{tabular}{|c|c|c|c|c|c|c|c|c|c|}
\hline \multirow[b]{2}{*}{ sitt } & & & ل & & \multicolumn{5}{|c|}{${ }^{*} \operatorname{gg} \mathrm{G}, \operatorname{IgM}$ and IgA serology sampling } \\
\hline & $7^{\star}$ & $15^{*}$ & $21^{*}$ & $28^{*}$ & $35^{\star}$ & $42^{*}$ & $56^{*}$ & $77^{\star}$ & 98 \\
\hline & & $\begin{array}{l}\downarrow \\
\text { T ce } \\
\text { prol }\end{array}$ & $\begin{array}{l}\downarrow \\
\text { Sple } \\
\text { NAL }\end{array}$ & & ytokin & & $\begin{array}{l}\downarrow \\
\text { BM ASC } \\
\text { T cell prolif. }\end{array}$ & $\begin{array}{l}\downarrow \\
\text { IgG Isotypes }\end{array}$ & $\stackrel{\downarrow}{\text { Challenge }}$ \\
\hline
\end{tabular}
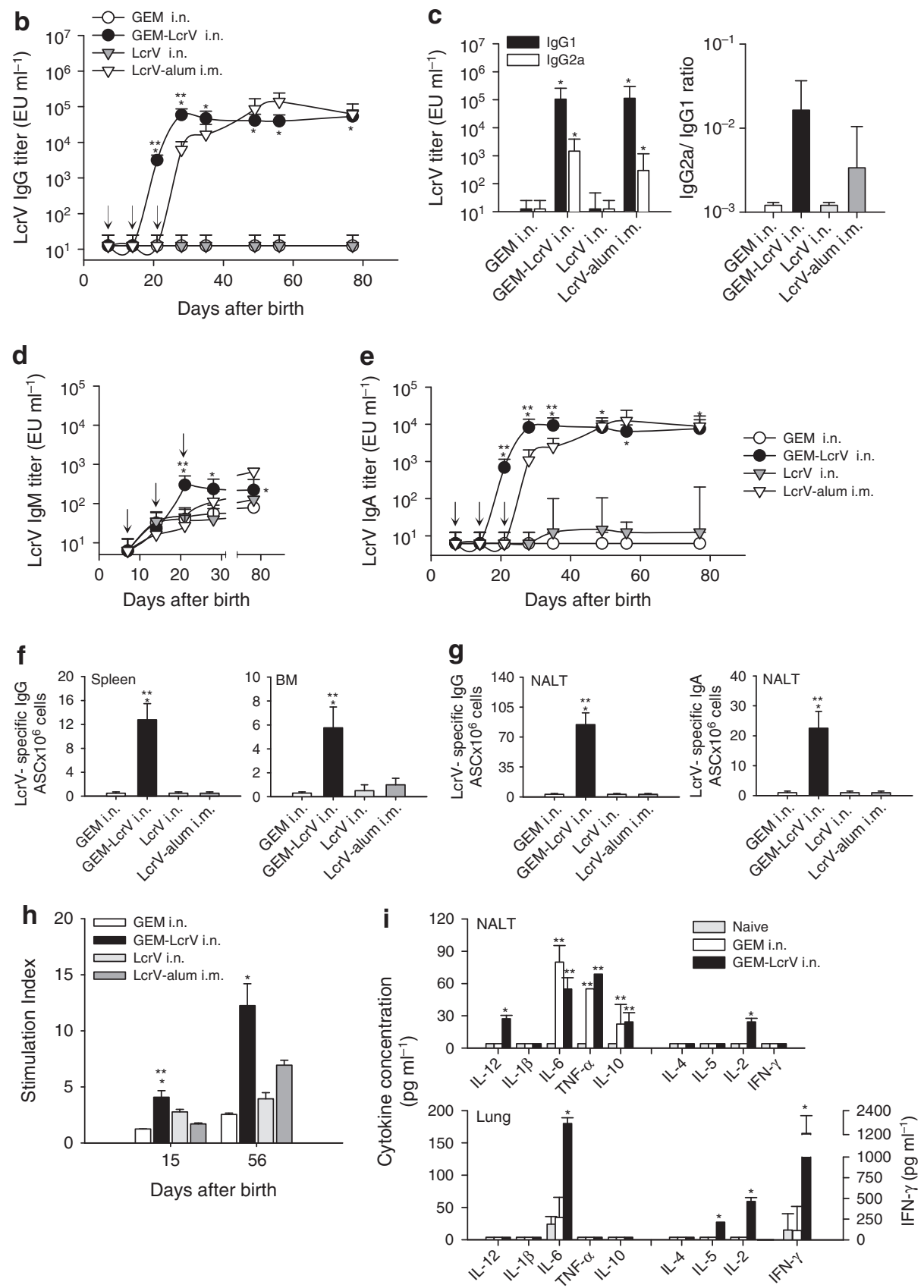

Figure 5 Intranasal immunization of newborn mice with L. lactis GEM-LcrV elicited potent systemic and mucosal immune responses. (a) Timeline for immunization and analysis of immune responses. Newborn mice (1-week-old, $n=21-28$ pups per group) were immunized i.n. with GEM-LcrV (carrying $\sim 12 \mu \mathrm{g}$ of $\mathrm{LcrV})$, GEMs alone or recombinant $\mathrm{LcrV}(20 \mu \mathrm{g})$ on days 7,15 , and 21 after birth (arrows). A positive control group received $2 \mu \mathrm{g}$ of $\mathrm{LcrV}$-alum i.m. (b) Kinetics of LcrV-specific serum IgG levels. (c) IgG subclass distribution (IgG1 and IgG2a titers) and IgG2a/lgG1 ratio. (d) Kinetics of LcrV-specific $\operatorname{lgM}$ and (e) IgA production. (b-e) Data represent median titers and ratios \pm one quartile. (f) LcrV-specific IgG ASC in the spleen and BM measured on days 21 and 56 after birth, respectively. (g) IgG and IgA ASC measured in the NALT 21 days after birth. Results shown are the mean ASC frequency per $1 \times 10^{6}$ cells \pm S.e.m. of replicate wells. (h) Proliferation of LcrV-specific T cells measured by $\left[{ }^{3} \mathrm{H}\right]$ thymidine incorporation. Results are expressed as stimulation index \pm s.e.m. from replicate wells. (b-h) Significant differences compared with newborn mice that received GEM alone $\left({ }^{\star} P<0.002\right)$ or LcrV-alum i.m. $\left({ }^{\star \star} P<0.01\right)$ are indicated. (i) Cytokine profile in the NALT and lungs measured on day 21 after birth. Cells were restimulated in vitro with LcrV. Data represent mean cytokine levels \pm s.d. from triplicate cultures. Significant differences compared with GEMs alone $\left({ }^{\star} P<0.001\right)$ and naive mice $\left({ }^{\star *} P<0.005\right)$ are indicated. ASC, antibody secreting cell; BM, bone marrow; GEM, Gram-positive enhancer matrix; NALT, nasal-associated lymphoid tissue. 

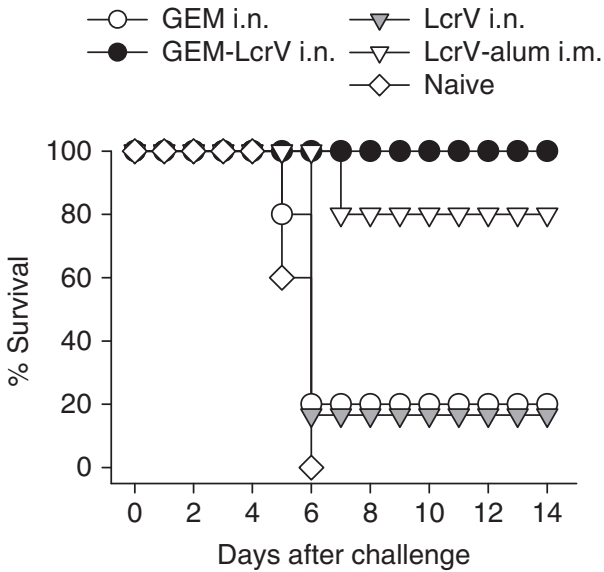

Figure 6 Protection against systemic lethal plague infection. Newborn mice ( $n=5-6$ pups per group) were immunized as described above. Eight weeks after the last immunization they were challenged intravenously with Y. pestis EV76 $\left(\sim 30 \mathrm{MLD}_{50}\right)$ in the presence of $\mathrm{FeCl}_{2}$. Data show percent survival during the monitoring period. GEM, Gram-positive enhancer matrix.

these groups showed significant weight loss and signs of disease. Among those immunized i.m. with LcrV-alum, $80 \%$ were protected. These animals showed moderate signs of disease during the first week but recovered thereafter.

\section{Human DCs stimulated with GEM particles showed an activated and mature phenotype}

To further evaluate the potential of the L. lactis GEM particles as a vaccine platform for human newborns, we investigated whether the immune stimulatory properties observed in mice also extended to human neonatal DCs. Human newborn DCs derived from $\mathrm{CD} 34^{+}$precursors from umbilical cord blood were stimulated with GEM particles or were mock treated. DC derived from peripheral mononuclear cells from adult human subjects were tested in parallel to compare the degree of activation of neonatal vs. adult DC. In both cases, stimulation with TNF- $\alpha$ was included as a positive control. The expression of activation and maturation markers on $\mathrm{CD} 11 \mathrm{c}^{+}$DCs was measured by flow cytometry. GEM-stimulated neonatal and adult human DC upregulated expression of MHC-II (HLA-DR) and costimulatory molecules CD80, CD86, and CD83 (Figure 7a). Consistent with the results obtained in mice, CD86 showed the highest increase in neonatal DCs, whereas both CD86 and HLADR were the most increased in adult cells (Table 1). The ability of human newborn CD11 ${ }^{+}$DC to phagocytose the GEM particles was demonstrated by confocal laser microscopy (Figure 7b). A cluster of FITC-stained GEM particles can be seen in the DC cytoplasm, and the abundant red surface staining indicates CD11c expression.

\section{Human DC matured by GEM particles had decreased phagocytic activity and enhanced capacity for cytokine secretion and T-cell activation}

The functional capacity of GEM-stimulated neonatal and adult human $\mathrm{CD} 11 \mathrm{c}^{+} \mathrm{DC}$ was assessed by measuring the levels of
Th1-promoting, proinflammatory and T-regulatory cytokines in culture supernatant of cells treated with GEMs or TNF- $\alpha$ (positive control), or mock-treated cells. Increased levels of TNF- $\alpha$, IL-10, IL-6, and IL-8 were produced by both neonatal and adult human DCs. Production of IL-12p70 was also elevated in neonatal DC, and production of IL- $1 \beta$ was elevated in adult DCs (Figure 7c). Consistent with their activated and mature phenotype, human neonatal and adult DCs stimulated with GEM particles showed reduced phagocytic activity, as evidenced by lower levels of FITC-dextran uptake compared with mocktreated DCs (Figure 7d).

Finally, we examined the capacity of the GEM-activated human neonatal and adult DCs to stimulate $\mathrm{CD}^{+} \mathrm{T}$ cells in a mixed lymphocyte reaction (Figure 7e). TNF- $\alpha$ - and mock-treated DCs were included as controls. Mature DCs expressing high levels of MHC-II molecules, as opposed to immature DC, are expected to activate allogeneic T cells. Indeed, proliferative responses were higher when $\mathrm{T}$ cells were stimulated with GEM-activated DCs compared with mocktreated DCs. T-cell activation followed a dose-response pattern, with proliferation levels increasing in the presence of higher DC/T cell ratios.

\section{DISCUSSION}

In this study, we demonstrated the usefulness of non-living and non-genetically modified $L$. lactis antigen-displaying particles for early life immunization. We show, for the first time, that intranasal immunization of newborn mice with $L$. lactis GEMs displaying $Y$. pestis $\mathrm{LcrV}$ is well tolerated and elicits a potent mucosal and systemic immunity that protects against lethal plague infection.

The GEM particles accomplished one of the most desired tasks for a neonatal vaccine: they effectively targeted a vaccine antigen into neonatal DC, and activated and enhanced the maturation of these cells in such a manner that they became proficient antigen-presenting cells capable of stimulating antigen-specific Th1type effector cells. Both neonatal mouse and human DCs were powerfully activated and acquired a mature phenotype when exposed to the GEMs. Neonatal DCs from mucosal lymphoid tissues also acquired a mature phenotype when stimulated by the GEM particles in vivo.

Newborn mice immunized i.n. with GEMs displaying $Y$. pestis LcrV induced high levels of LcrV-specific serum antibodies, systemic and mucosal IgG and IgA ASCs, and robust T-cell responses. LcrV was remarkably immunogenic when carried by the GEM particles but not when given alone, which is probably the result of higher efficiency of key processes, including the activation of innate immunity through interaction with TLR2, DCs uptake, and direct stimulation of B cells. A proinflammatory cytokine milieu with elevated levels of IL-6 and TNF- $\alpha$, indicative of a general immune stimulation, was found in the NALT of newborns immunized with the GEMs. IL-10 was also produced, most likely to counter-balance such broad immune activation. The excellent tolerability of the vaccine also indicates that a finely tuned mechanism of immune balance indeed takes place. 
a

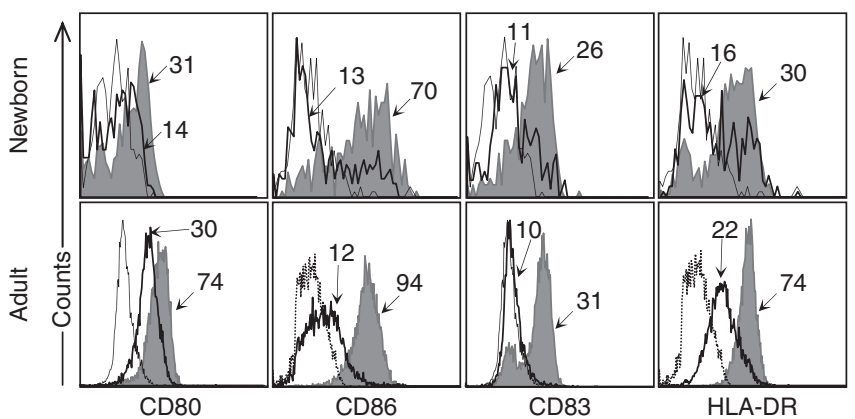

b

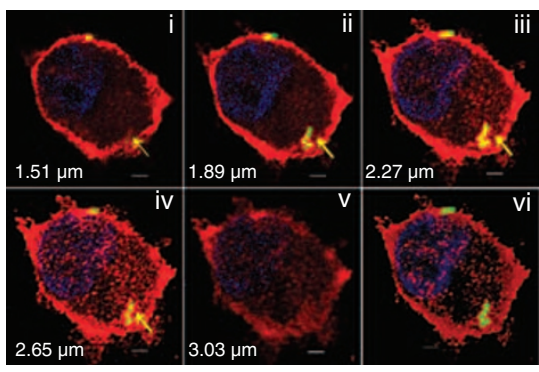

Red, CD11c; green, GEMs; blue, nuclei
C

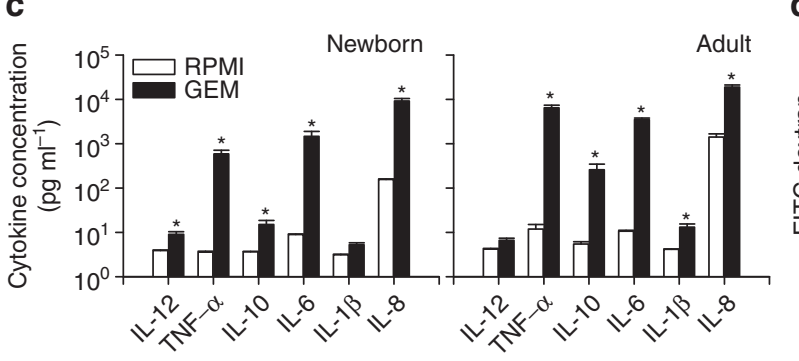

d
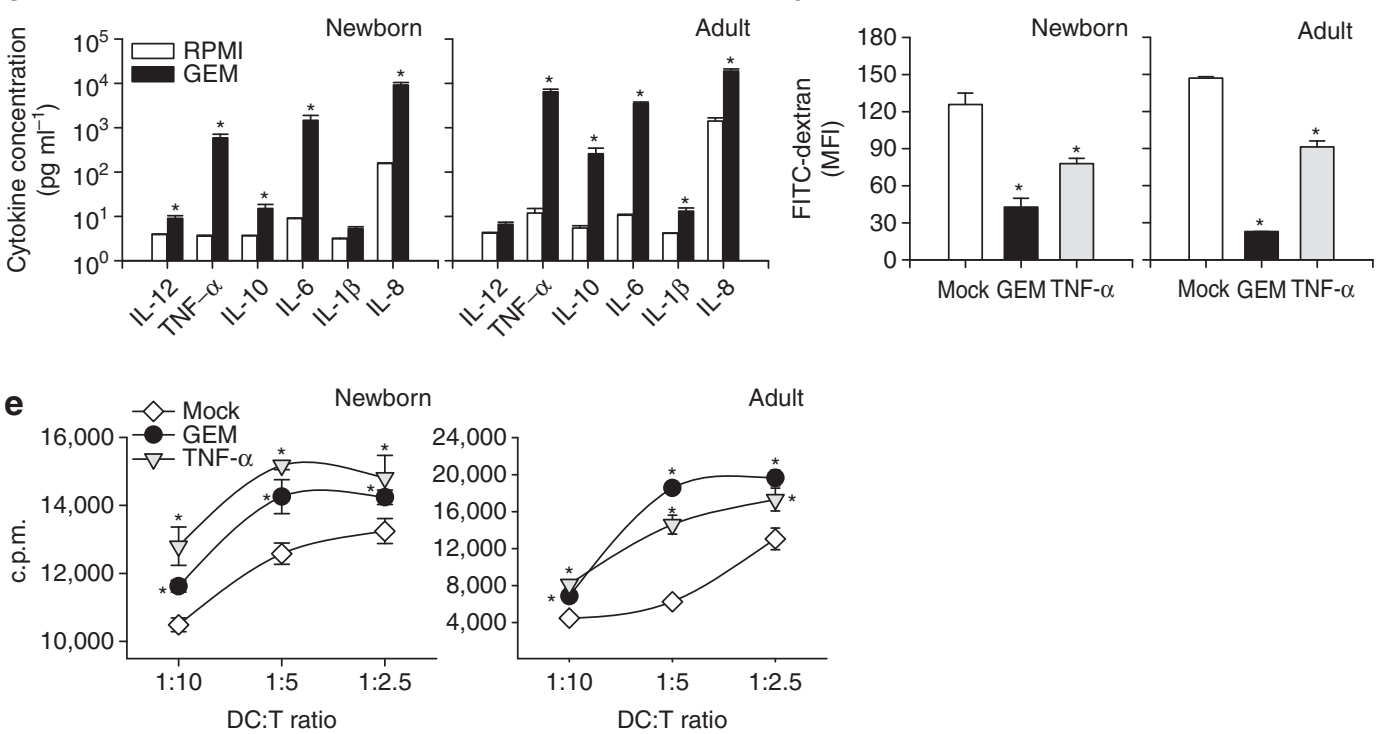

Figure 7 The GEM particles enhanced the maturation of neonatal and adult human DC and the stimulation of T cells in an MLR. (a) Expression of cell-surface markers CD80, CD86, CD83, and HLA-DR on human CD11 $\mathrm{c}^{+}$neonatal and adult DC stimulated with GEM particles (shaded area) or mock-treated (solid line); the dashed line indicates the isotype control. The MFI on CD11 $\mathrm{c}^{+}$gated cells is indicated. (b) Individual (i-v) and Z-stack projection (vi) confocal laser microscopy images showing CD11 $\mathrm{c}^{+} \mathrm{DC}$ from human newborns harboring FITC-labeled GEM particles (arrows). CD11 ${ }^{+}$ cell-surface expression is shown in red, and nuclei are shown by blue fluorescent staining. Bar $=2 \mu \mathrm{m}$. (c) Cytokines produced by human neonatal $\mathrm{CD}_{3}{ }^{+}$- and adult-derived DC stimulated with GEM particles or mock-stimulated and measured in culture supernatants. Data represent mean cytokine concentration \pm S.e.m. from two independent experiments. (d) FITC-dextran uptake by neonatal CD11 ${ }^{+}$and adult human DC exposed to GEM or TNF- $\alpha$ (positive control), or mock-treated DC, measured by flow cytometry; data represent MFI \pm s.e.m. from three independent experiments. (e) Allogeneic stimulation of adult $\mathrm{CD}^{+} \mathrm{T}$ cells in the presence of neonatal and adult human DC stimulated with GEM particles or TNF- $\alpha$, or mock-stimulated DC. Data show mean cpm \pm s.e.m. from one of two independent experiments. (c-e) Significant differences $\left({ }^{\star} P<0.05\right)$ compared with mock-stimulated cells. DC, dendritic cell; GEM, Gram-positive enhancer matrix; HLA, human histocompatibility leukocyte antigen; MFI, mean fluorescence intensity; MLR, mixed lymphocyte reaction, TNF, tumor necrosis factor.

Neonatal mucosal immunization with GEM-LcrV also surpassed parenteral immunization with LcrV-alum in (i) the swiftness to reach peak antibody level, (ii) the steadiness of the antibody response, (iii) the capacity to induce mucosal and systemic ASCs, and (iv) the ability to prime systemic effector T cells early in life. The induction of potent immune responses in the respiratory mucosa is particularly important, as this is the portal of entry for many of the pathogens that cause severe disease in early childhood. Such a response can block infection locally, preventing systemic dissemination of the pathogen. Earlier studies have shown mucosal and systemic responses in adult mice immunized i.n. with GEM particles displaying pneumococcal antigens ${ }^{16}$ and significant protection against lethal respiratory pneumococcal challenge. ${ }^{17}$ The capacity of the GEM-LcrV particles to induce high levels of antigen-specific IFN- $\gamma$ and IL- 2 in the lungs is a remarkable finding considering the importance of Th1-type responses in the protection of young hosts against intracellular pathogens and the difficulty of inducing such effector responses early in life, especially in a mucosal tissue. Most importantly for its use as a prophylactic vaccine, intranasal immunization with GEM-LcrV conferred the highest level of protection against lethal $Y$. pestis infection in neonatally immunized mice. The capacity of the GEMs to activate nonspecific immune defenses can explain the $20 \%$ survival in mice that received particles alone, as opposed to complete lethality in the naive group. This effect is most likely due to the PGN (exposed on the particle surface), which has been shown to stimulate not only innate immune cells but also $\mathrm{T}$ cells, including those of 
neonatal origin, through interaction with TRL2. ${ }^{18-20}$ Despite this broad immune activation, the protection conferred by the GEM-LcrV was clearly antigen-specific.

Yersinia pestis causes a severe and often fatal disease in humans for which no licensed vaccine is available. ${ }^{15}$ The pulmonary form (pneumonic plague) is particularly virulent as it quickly spreads from person to person through aerosol droplets. ${ }^{21}$ Y. pestis has drawn considerable attention lately as a potential biological weapon, and biowarfare concerns have revived efforts toward the development of new vaccine candidates. These endeavors, however, have been focused almost exclusively on vaccines for adults, and these vaccines are unlikely to meet the needs of other (more vulnerable) segments of the population, such as children. A $L$. lactis GEM-based plague vaccine for pediatric use would represent a unique tool in the biodefense armamentarium. If needed, the GEM-LcrV platform could be further improved by displaying additional $Y$. pestis antigens such as F1 or Yersinia outer proteins (Yops). A GEM-based plague vaccine would be ideal for early life, surpassing the leading alum-adjuvanted F1-V vaccine in the quality and strength of the responses and surpassing live attenuated vaccine strains with regard to safety.

Recombinant lactic acid bacteria, including L. lactis strains, expressing heterologous antigens have been sought as mucosal vaccines and could potentially be used in children. ${ }^{22-25}$ Major concerns associated with the use of these organisms include the possibility of causing disease by establishing niches within the commensal flora and the introduction of exogenous DNA, especially plasmids that confer antibiotic resistance. The GEM particles alleviate these concerns because they are non-living and non-genetically modified. They also have many other advantages over live recombinant vectors that increase their appeal for infant immunization: (i) the amount of antigen displayed and delivered in vivo is known and remains constant, unlike antigen expression in living recombinant organisms, which can be affected by plasmid loss, metabolic burden and promoter activation; (ii) the antigen is always exposed on the particle surface at a high density and in a multimeric form, facilitating the activation of B cells; (iii) multiple antigens can be displayed simultaneously, a feature that has not yet been achieved using live probiotics; and (iv) the GEMs are highly stable and can be stored at room temperature, avoiding the need of cold chain.

The GEMs have additional properties that make them suitable for large-scale vaccination in field settings: their production is simple and inexpensive, and they are easy to preserve and to administer. A recent structural analysis of GEM-LcrV particles showed that the attachment of the LcrV-PGN binding domain fusion proteins to the particle surface is indeed strong, stable, specific (proteins without the PGN anchor domain do not bind to the particles), and abundant (multiple antigen clusters are loaded onto the surface) ${ }^{26}$ It is also $\mathrm{pH}$ specific: $\mathrm{LcrV}$ binds to the particles under physiological conditions ( $\mathrm{pH} 7.2$ ), but detaches when exposed to an acidic environment ( $\mathrm{pH} 4.4$ ), facilitating its release within the phagolysosomes for processing and presentation. ${ }^{26}$

The $L$. lactis GEMs were administered i.n. to newborn mice with the rationale that being inert particles, L. lactis GEMs could also conceivably be given to human infants by the nasal route, thus departing from the traditional orogastric delivery of live probiotics. There are also many advantages associated with intranasal immunization. Vaccine antigens administered by this route are less likely to be blocked by maternal antibodies. ${ }^{27,28}$ Intranasal vaccination is not affected by physiological intestinal barriers, including the resident flora, which may prevent access of vaccine antigens to immunological inductive sites. Nor does intranasal vaccination disturb the gastrointestinal microbial environment, which has an important role in the process of neonatal immune development. ${ }^{29}$ Furthermore, a nasally delivered vaccine would encounter a higher frequency of "immunogenic" as opposed to "tolerogenic" DCs, more prevalent in the gut.

A handful of studies have examined the ability of L. lactis to activate mouse and human DC in vitro. ${ }^{17,23,30-33}$ These studies described the phenotype of the activated cells but, for the most part, did not examine their capacity for immunological priming. We demonstrated the ability of "mature" GEM-stimulated neonatal DCs to present a vaccine antigen and to stimulate Th1type effector cells in a neonatal setting. Our results agree with those of Niers et al., ${ }^{32}$ who showed in vitro that naive neonatal human $\mathrm{T}$ cells can be skewed toward a Th1-type response upon exposure to L. lactis-primed DCs.

This is the first report seeking the use of probiotic-derived antigen-displaying particles to "jump-start" the neonatal immune system for the purpose of early life immunization. Also unique to our studies is the comparison of vaccine-activated neonatal and adult DCs from both mice and humans, demonstrating in both cases that the GEM particles can induce neonatal DC activation levels comparable with those achieved by adult DCs.

In summary, we demonstrated that L. lactis GEM particles have the inherent capacity to activate the neonatal immune system and that they provide a well tolerated, effective, and practical vaccine platform for early life immunization, overcoming the limitations of conventional vaccines. These results show the exceptional potential of GEM-based vaccines and warrant future studies of such vaccines in humans.

\section{METHODS}

Generation of neonatal and adult DCs. Dendritic cells were isolated from the BM of newborn (7-day-old) and adult (6- to 8-weekold) $\mathrm{BALB} / \mathrm{c}$ mice as previously described. ${ }^{34}$ One-week-old newborn mice were used in all experiments, as their state of immune maturation is considered equivalent to that of human neonates. ${ }^{1}$ Cells were cultured in RPMI 1640 containing $2 \mathrm{~mm}$ L-glutamine, $10 \mathrm{~mm}$ HEPES (Gibco-Invitrogen, Grand Island NY), 0.1 mm 2-mercaptoethanol (Sigma-Aldrich, St Louis, MO), $50 \mu \mathrm{g} \mathrm{ml}^{-1}$ gentamicin and $10 \%$ fetal bovine serum (HyClone, Logan, UT) (cRPMI) for 7 days in the presence of granulocyte-macrophage-colony-stimulating factor and IL-4 $\left(10 \mathrm{ng} \mathrm{ml}^{-1}\right)$ (PeproTech, Rocky Hill, NJ) at $37^{\circ} \mathrm{C}, 5 \% \mathrm{CO}_{2}$. CD $11 \mathrm{c}^{+}$ DCs were further purified by negative magnetic cell sorting (BD Pharmingen, San Jose, CA); purity ranged between 80 and 95\%. Human cord blood was obtained from the placentas of normal, full-term deliveries at the University of Maryland Medical Center. DCs were generated from magnetically sorted $\mathrm{CD} 34^{+}$progenitors (Miltenyi Biotec, Auburn, CA). Adult peripheral blood samples were obtained from healthy volunteers, and mononuclear cells were isolated by density 
gradient centrifugation with lymphocyte separation medium (LSM, MP Biomedicals, Ontario, Canada). Cells were allowed to adhere during $3 \mathrm{~h}$ at $37^{\circ} \mathrm{C}, 5 \% \mathrm{CO}_{2}$. Adult adherent and magnetically sorted cord blood CD $34^{+}$cells were cultured for 7 days in cRPMI with $50 \mathrm{ng} \mathrm{ml}^{-1}$ of human granulocyte-macrophage-colony-stimulating factor and $10 \mathrm{ng} \mathrm{ml}^{-1}$ of IL-4 (Peprotech). Blood collection was approved by the University of Maryland Institutional Review Board.

DC stimulation and flow cytometry. $\mathrm{CD} 11 \mathrm{c}^{+} \mathrm{DCs}$ from adult and newborn mice were cultured for $24 \mathrm{~h}$ in the presence of GEM particles (1:150 DC/GEM), E. coli LPS ( $5 \mathrm{ng} \mathrm{ml}^{-1}$, Sigma-Aldrich, St Louis, MO) as positive control or cRPMI (mock-treated). Following incubation, the cells were washed and stained with FITC-, PE-, or allophycocyanin-labeled monoclonal antibodies specific for CD40, CD80, MHC II, CD86, and CD11c in PBA (phosphate-buffered saline (PBS) containing $0.1 \%$ bovine serum albumin and $\left.0.01 \% \mathrm{NaN}_{3}\right) \cdot{ }^{34} \mathrm{Fc}$-Block (anti-mouse CD16/32) was used to reduce background staining. In studies of in vivo DC activation, mononuclear cells from nasal and lung tissues were collected from 15day-old mice that had been immunized with GEM particles (or mock immunized) and stained as described above. Human DCs were cultured for $24 \mathrm{~h}$ with GEMs (1:150), human TNF- $\alpha$ ( $5 \mathrm{ng} \mathrm{ml}^{-1}$, PeproTech), or cRPMI (mock-treated). Cells were stained with FITC-, PE-, allophycocyanin, or PerCP Cy5.5-labeled monoclonal antibodies specific for CD1a, CD80, CD83, CD86, CD11c, and HLA-DR (BD Pharmingen). Fc $\gamma$ R-binding inhibitor (eBioscience, San Diego, CA) was used to reduce background straining. Stained cells were fixed with $4 \%$ paraformaldehyde and run on a DakoCytomation MoFlo flow cytometer (Carpinteria, CA). Data were collected from 10,000 to 30,000 cells and were analyzed using WinList 6.0 3D software (Verity Software House, Topsham, ME).

DC cytokine production. Cytokines were measured in 24-h culture supernatants from GEM- and control-treated DCs using the Cytometric Bead Array mouse and human proinflammatory cytokine kits (BD Pharmingen, Miami, FL). Samples were run on a Beckman Coulter Epics Elite flow cytometer. Cytokine concentrations were determined using standard reference curves using Cytometric Bead Array software (BD Pharmingen).

FITC-dextran uptake. Mouse and human $\mathrm{CD} 11 \mathrm{c}^{+}$cells treated with GEMs or mock-treated were incubated with $1 \mathrm{mg} \mathrm{ml}^{-1}$ FITC-dextran (Sigma-Aldrich) for $1 \mathrm{~h}$ at 37 or $4^{\circ} \mathrm{C}$. Cells were washed with cold PBA buffer and analyzed by flow cytometry. Dextran uptake was measured as the increase in the number of FITC-positive cells incubated at 37 vs. $4^{\circ} \mathrm{C}$.

TLR signaling. Toll-like receptor stimulation was assessed in HEK293 cells expressing human TLR2, 3, 4, 5, 7, 8, or 9 or expressing mouse TLR7 or TLR9 (one TLR at a time). The activity of alkaline phosphatase, which was under the control of nuclear factor- $\kappa \mathrm{B}$-inducible promoter, was measured as a readout for TLR activation. The assays were performed at InvivoGen (San Diego, CA). Briefly, cells were incubated for $16-20 \mathrm{~h}$ with $20 \mu \mathrm{l}$ of GEMs $\left(\sim 5 \times 10^{8}\right.$ particles $)$ or positive control ligands as follows: hTLR2, heat-killed Listeria monocytogenes $\left(10^{8}\right.$ cells $\left.\mathrm{ml}^{-1}\right)$; hTLR3, Poly(I:C) $\left(1 \mu \mathrm{g} \mathrm{ml}^{-1}\right)$; hTLR4, E. coli K12 LPS (100 $\left.\mathrm{ng} \mathrm{ml}^{-1}\right)$; hTLR5, Salmonella typhimurium flagellin $\left(100 \mathrm{ng} \mathrm{ml}^{-1}\right)$; hTLR7, gardiquimod $\left(1 \mu \mathrm{g} \mathrm{ml}^{-1}\right)$; hTLR8, CL075 $\left(1 \mu \mathrm{g} \mathrm{ml}^{-1}\right)$; hTLR9, CpG ODN $2006\left(100 \mathrm{ng} \mathrm{ml}^{-1}\right)$; mTLR7, gardiquimod $\left(1 \mu \mathrm{g} \mathrm{ml}^{-1}\right)$; and mTLR9, CpG ODN $1826\left(1 \mu \mathrm{g} \mathrm{ml}^{-1}\right)$. Cells incubated with TNF- $\alpha\left(100 \mathrm{ng} \mathrm{ml}^{-1}\right)$ were included as a positive control for nuclear factor- $\kappa \mathrm{B}$ activation. Absorbance at $650 \mathrm{~nm}$ was measured in an enzyme-linked immunosorbent assay reader.

TLR2 neutralization. Newborn or adult mouse BM-derived magnetically sorted CD $11 \mathrm{c}^{+}$DCs were preincubated with $20 \mu \mathrm{g} \mathrm{ml}^{-1}$ of anti-human and mouse TLR2 monoclonal antibody (eBioscience) or isotype control ( $1 \mathrm{~h}$ at room temperature) and were then cultured for $24 \mathrm{~h}$ with GEM particles (1:150) or cRPMI (mock-treated). IL-6 released to the culture supernatants was measured by enzyme-linked immunosorbent assay (eBioscience).

Vaccines. Lactococcal GEM-LcrV particles were prepared as follows: the $l c r V$ gene from $Y$. pseudotuberculosis (identical to the LcrV gene of $Y$. pestis strain KIM5, nt 481-1461 of GenBank entry \#M26405) was subcloned by PCR from plasmid pSEC91-LcrV, and the amplified $l c r V$ gene fragment was cloned into plasmid pPA3. ${ }^{35}$ The correct insertion of the gene was verified by DNA sequencing. The resulting plasmid, pPA281, was electroporated into L. lactis PA1001 for expression and secretion of the recombinant LcrV-PGN anchor domain fusion protein, as previously described. ${ }^{9}$ The lactococcal PGN binding domain enables strong non-covalent binding of the vaccine antigen to GEM particles. ${ }^{8}$ GEM particles prepared by heat-acid treatment of $L$. lactis were mixed with LcrV-PGN binding domain fusions for $30 \mathrm{~min}$ at room temperature. ${ }^{8,9}$ One vaccine dose for GEM and GEM-LcrV contained $2.5 \times 10^{9}$ particles. The amount of LcrV bound to the GEM particles was determined by densitometry analysis; $2.5 \times 10^{9}$ particles contained $20 \mu \mathrm{g}$ of LcrV-PGN fusion protein. The LcrV fragment represents about $60 \%$ of the fusion protein, meaning that $12 \mu \mathrm{g} \mathrm{LcrV}$ was present in each vaccine dose. The particles were centrifuged and resuspended in enough sterile PBS to obtain the desired GEM-LcrV concentration before immunization. Recombinant full-length Y. pestis LcrV (without the PGN binding domain) purified from $E$. coli was used as subunit vaccine for nasal and parenteral immunization. Residual LPS was removed using Detoxi-Gel columns (Thermo Scientific, Rockford, IL) and endotoxin levels $\left(<0.19 \mathrm{EU} \mathrm{mg}^{-1}\right) \mathrm{moni}-$ tored by chromogenic LAL test (Lonza, Allendale, NJ).

Fluorescence microscopy. GEM-LcrV and GEM particles were stained with mouse anti-LcrV monoclonal antibody (AbCam, Cambridge, MA) or an isotype control in PBA for $1 \mathrm{~h}$ at room temperature, followed by staining with FITC-labeled anti-mouse IgG (Invitrogen, Carlsbad, CA) for $30 \mathrm{~min}$. The particles were washed, mounted in Prolong Gold (Invitrogen) and visualized using a Nikon Eclipse 2000-E UV fluorescence microscope (Melville, NY). Images were acquired using MetaVue software, version 6.1 (Universal Imaging, Downingtown, PA).

In vitro antigen presentation. Negatively sorted CD11c ${ }^{+} \mathrm{DCs}$ from newborn mice were cultured for $24 \mathrm{~h}$ with GEMs alone, GEM-LcrV (1:100), LcrV $\left(1.8 \mu \mathrm{g} \mathrm{ml}^{-1}\right)$, or cRPMI (mock-stimulated). Cells were washed, irradiated and co-cultured with $\mathrm{CD} 4^{+}$and $\mathrm{CD} 8^{+} \mathrm{T}$ cells (90-95\% purity) obtained from the spleen of naive and LcrV-immune adult mice by magnetic sorting (Dynal Biotech, Oslo, Norway). This last group had been immunized twice with $2 \mu \mathrm{g}$ of LcrV-alum i.m., 14 days apart, and spleens were harvested 3 weeks after the last immunization. Increasing numbers of purified CD $11 c^{+}$DC were co-cultured with $1 \times 10^{5}$ naive or $\mathrm{LcrV}$-specific $\mathrm{CD} 4^{+}$and $\mathrm{CD} 8^{+} \mathrm{T}$ cells. As readouts of T-cell stimulation, we measured lymphocyte proliferation by $\left[{ }^{3} \mathrm{H}\right]$ thymidine incorporation and the frequency of IFN- $\gamma$-secreting cells by ELISPOT, as previously described. ${ }^{34} \mathrm{CD} 4^{+}$and $\mathrm{CD} 8^{+} \mathrm{T}$ cells alone, $\mathrm{CD} 11 \mathrm{c}^{+} \mathrm{DC}$ alone and $\mathrm{CD} 11 \mathrm{c}^{+} \mathrm{DC}$ cultured with naive $\mathrm{CD} 4^{+}$ and $\mathrm{CD}^{+} \mathrm{T}$ cells were included as negative controls and their responses were subtracted from experimental wells. ConA $\left(2 \mu \mathrm{g} \mathrm{ml}^{-1}\right.$, Sigma $)$ or $\operatorname{LcrV}\left(5 \mu \mathrm{g} \mathrm{ml}^{-1}\right)$ served as positive controls.

Mice and immunization. BALB/c mice (8- to 10 -week-old, Charles River Laboratories, Wilmington, MA) were bred and housed under specific pathogen-free conditions as previously described. ${ }^{36}$ Experimental groups contained three to five litters ( 6 pups per litter). Newborn mice were immunized i.n. on days 7, 15 and 21 after birth with 1 dose $\left(2.5 \times 10^{9}\right.$ particles $)$ of GEM-LcrV $(12 \mu \mathrm{g} \mathrm{LcrV})$, with GEMs alone or with $20 \mu \mathrm{g}$ of recombinant $\mathrm{LcrV}(\sim 5 \mu \mathrm{l}, 2.5 \mu \mathrm{l}$ per nare). A group immunized i.m. on days 7, 15, and 21 after birth with $2 \mu \mathrm{g}$ of LcrV adsorbed to $0.5 \%$ alhydrogel (Brenntag Biosector, Frederikssund, Denmark) was included as a positive control. Blood samples were obtained at different time points, as previously described. ${ }^{34}$ Studies 
were approved by the University of Maryland Institutional Animal Care and Use Committee.

LcrV-specific antibodies. LcrV-specific serum IgG, IgM, and IgA levels were measured by enzyme-linked immunosorbent assay. Immulon II plates were coated with endotoxin-free $\mathrm{LcrV}$ at $1 \mu \mathrm{g} \mathrm{ml}^{-1}$ in carbonate buffer ( $\mathrm{pH}$ 9.6) for $3 \mathrm{~h}$ at $37^{\circ} \mathrm{C}$ and blocked overnight with $10 \%$ dry milk (Nestle, Solon, $\mathrm{OH}$ ) in PBS. Sera were tested in serial dilutions in PBS containing Tween 20 and 10\% dry milk. Antibodies were detected with horseradish peroxidase-labeled antibodies specific for mouse IgG, IgG1, IgG2a (Roche, Indianapolis, IN), IgM (Kirkegaard \& Perry Laboratories, KPL, Gaithersburg, MD), and IgA (Zymed, San Francisco, CA) followed by the TMB substrate (KPL). After $15 \mathrm{~min}$ of incubation, the reaction was stopped with $1 \mathrm{M} \mathrm{H}_{3} \mathrm{PO}_{4}$. End point titers were calculated through a linear regression as the reciprocal of the serum dilution that produced an absorbance $450 \mathrm{~nm}$ value of 0.2 above the blank and reported in enzymelinked immunosorbent assay units (EU) $\mathrm{ml}^{-1}$.

IgG and IgA ASC. Single-cell suspensions from the spleen, BM, and NALT collected on days 15, 21, and 56 after birth, as previously described, ${ }^{34}$ were incubated overnight at $37^{\circ} \mathrm{C}, 5 \% \mathrm{CO}_{2}$ in plates previously coated with $\operatorname{LcrV}\left(5 \mu \mathrm{g} \mathrm{ml}^{-1}\right)$ and blocked with cRPMI. Horseradish peroxidase-labeled goat anti-mouse IgG or IgA (Zymed) diluted in PBS with $1 \%$ bovine serum albumin were used as conjugates in agarose overlay. True Blue was used as the substrate (KPL). Spots were counted in a stereomicroscope, and the results were expressed as the mean number of IgG ASC per $10^{6}$ cells from quadruplicate wells. Spots from control wells were subtracted from experimental wells. A positive response was defined as $\geqslant 4$ spots per $10^{6}$ cells.

Cytokines in mucosal tissues. Nasal-associated lymphoid tissue and lung mononuclear cells were obtained on day 21 after birth from naive, GEM-, and GEM-LcrV-immunized newborn mice, as previously described. ${ }^{36}$ Cells were stimulated in vitro for $48 \mathrm{~h}$ with $\mathrm{LcrV}\left(2.5 \mu \mathrm{g} \mathrm{ml}^{-1}\right)$ or cRPMI (mock-stimulated), and cytokine levels in the culture supernatants were measured using the multiplex Meso Scale Discovery (MSD, Gaithersburg, MD) kits, as recommended by the manufacturer. Plates were read using the MSD Sector Imager 2400.

T-cell proliferation. Single-cell suspensions from spleens were incubated with $2.5 \mu \mathrm{g} \mathrm{ml}^{-1}$ of LcrV, cRPMI, or ConA $\left(5 \mu \mathrm{g} \mathrm{ml}^{-1}\right.$, positive control) for 3-7 days at $37^{\circ} \mathrm{C}, 5 \% \mathrm{CO}_{2}$. Cell proliferation was measured by incorporation of $\left[{ }^{3} \mathrm{H}\right]$ thymidine, as previously described. ${ }^{34,36}$

Y. pestis challenge. Mice were challenged intravenously with $\sim 30$ $\mathrm{MLD}_{50}\left(3.36 \times 10^{3}\right.$ colony-forming units) of $Y$. pestis EV76 in $0.2 \mathrm{ml}$ of sterile $\mathrm{PBS}$. $\mathrm{FeCl}_{2}$ ( $40 \mu \mathrm{g}$ per mouse) was administered intraperitoneally immediately before challenge to enhance bacterial virulence. Health status, weight loss, and survival were monitored daily for 14 days. ${ }^{34}$ All survivors were humanely euthanized at the end of the monitoring period.

Laser confocal microscopy. Gram-positive enhancer matrix particles $\left(25 \times 10^{9}\right.$ particles) were incubated with $0.01 \mathrm{~g}$ FITC (Sigma) in $0.1 \mathrm{M}$ boric acid, $\mathrm{pH} 9.0$, for $60 \mathrm{~min}$ at room temperature in a shaker. Cord blood-derived CD $11 \mathrm{c}^{+}$DCs were cultured with FITC-GEMs (1:100), washed, blocked with human Fc $\gamma$ R-binding inhibitor and stained with a monoclonal anti-human CD11c antibody (BD Pharmingen) in PBA for $1 \mathrm{~h}$ at room temperature, followed by staining with Alexa Fluor 546-labeled anti-mouse IgG (Invitrogen). To visualize the nuclei, cells were incubated with 4',6'-diamidino-2-phenylindole dihydrochloride (DAPI, Invitrogen) in PBA buffer and were mounted in Prolong Gold (Invitrogen). Stained DCs were visualized using a Zeiss LSM510 META laser scanning confocal microscope (Göttingen, Germany). Images show the individual and Z-stack projections collected at $0.41 \mu \mathrm{m}$ intervals, analyzed with Zeiss LSM Examiner version 4.2 software.
Mixed lymphocyte reaction. Magnetically sorted adult human $\mathrm{CD}^{+}$ $T$ cells obtained from peripheral blood were cultured for 5 days with irradiated cord blood- or adult-derived CD $11 \mathrm{c}^{+}$DC pretreated with GEMs, human TNF- $\alpha$, or cRPMI (mock-treated). Negative controls included T cells and DC alone. T-cell proliferation was measured by $\left[{ }^{3} \mathrm{H}\right]$ thymidine incorporation.

Statistical analysis. Immune responses were compared using Student's $t$-test or the Mann-Whitney $U$-test. Differences with $P<0.05$ were considered significant. Statistical analysis was performed using SigmaStat 3.1 (SigmaStat software, Erkrath, Germany).

\section{ACKNOWLEDGMENTS}

We thank personnel from the CVD Applied Immunology Section for exceptional technical support, Dr Scott Lloyd (CVD) for providing plasmid pSEC91-LcrV, Dr Wilbur Chen and Dr Alan Cross (CVD) for TLR2/4 preliminary studies, and Dr Govert Schouten (Mucosis BV) for helpful discussions. This study was supported, in part, by the National Institute of Health Grant RO1-AI065760 (to MFP) and Mucosis BV.

\section{DISCLOSURE}

The authors declared no conflict of interest.

(C) 2010 Society for Mucosal Immunology

\section{REFERENCES}

1. Siegrist, C.A. Neonatal and early life vaccinology. Vaccine. 19, 3331-3346 (2001).

2. Siegrist, C.A. The challenges of vaccine responses in early life: selected examples. J. Comp. Pathol. 137 (Suppl 1), S4-S9 (2007).

3. Wilson, C.B. \& Kollmann, T.R. Induction of antigen-specific immunity in human neonates and infants. Nestle Nutr. Workshop Ser. Pediatr. Program. 61, 183-195 (2008).

4. Cortese, V.S. Neonatal immunology. Vet. Clin. North. Am. Food. Anim. Pract. 25, 221-227 (2009).

5. Willems, F., Vollstedt, S. \& Suter, M. Phenotype and function of neonatal DC. Eur. J. Immunol. 39, 26-35 (2009).

6. Adkins, B., Leclerc, C. \& Marshall-Clarke, S. Neonatal adaptive immunity comes of age. Nat. Rev. Immunol. 4, 553-564 (2004).

7. Demirjian, A. \& Levy, O. Safety and efficacy of neonatal vaccination. Eur. J. Immunol. 39, 36-46 (2008).

8. Bosma, T. et al. Novel surface display system for proteins on nongenetically modified Gram-positive bacteria. Appl. Environ. Microbiol. 72, 880-889 (2006).

9. van Roosmalen, M.L. et al. Mucosal vaccine delivery of antigens tightly bound to an adjuvant particle made from food-grade bacteria. Methods 38, 144-149 (2006).

10. Mshvildadze, M., Neu, J. \& Mai, V. Intestinal microbiota development in the premature neonate: establishment of a lasting commensal relationship? Nutr. Rev. 66, 658-663 (2008).

11. Guandalini, S. Probiotics for children with diarrhea: an update. J. Clin. Gastroenterol. 42 (Suppl 2), S53-S57 (2008).

12. Srinivasan, R., Meyer, R., Padmanabhan, R. \& Britto, J. Clinical safety of Lactobacillus casei shirota as a probiotic in critically ill children. J. Pediatr. Gastroenterol. Nutr. 42, 171-173 (2006).

13. Myhre, A.E., Aasen, A.O., Thiemermann, C. \& Wang, J.E. Peptidoglycan-an endotoxin in its own right? Shock 25, 227-235 (2006).

14. Ueno, $\mathrm{H}$. et al. Dendritic cell subsets in health and disease. Immunol. Rev. 219, 118-142 (2007).

15. Smiley, S.T. Current challenges in the development of vaccines for pneumonic plague. Expert. Rev. Vaccines 7, 209-221 (2008).

16. Audouy, S.A. et al. Lactococcus lactis GEM particles displaying pneumococcal antigens induce local and systemic immune responses following intranasal immunization. Vaccine 24, 5434-5441 (2006).

17. Audouy, S.A. et al. Development of lactococcal GEM-based pneumococcal vaccines. Vaccine 25, 2497-2506 (2007).

18. Yamamoto, S., Tin-Tin, W.S, Yoshida, Y., Kunugita, N., Arashidani, K. \& Fujimaki, H. Children's immunology, what can we learn from animal studies (2): modulation of systemic Th1/Th2 immune response in infant mice after prenatal exposure to low-level toluene and toll-like receptor (TLR) 2 ligand. J. Toxicol. Sci. 34 (Suppl 2), SP341-SP348 (2009). 
19. McCarron, M. \& Reen, D.J. Activated human neonatal CD8+ T cells are subject to immunomodulation by direct TLR2 or TLR5 stimulation. J. Immunol. 182, 55-62 (2009).

20. Parant, M., Parant, F. \& Chedid, L. Enhancement of the neonate's nonspecific immunity to Klebsiella infection by muramyl dipeptide, a synthetic immunoadjuvant. Proc. Natl. Acad. Sci. USA 75, 3395-3399 (1978).

21. Kool, J.L. Risk of person-to-person transmission of pneumonic plague. Clin. Infect. Dis. 40, 1166-1172 (2005).

22. Mohamadzadeh, M., Duong, T., Sandwick, S.J., Hoover, T. \& Klaenhammer, T.R. Dendritic cell targeting of Bacillus anthracis protective antigen expressed by Lactobacillus acidophilus protects mice from lethal challenge. Proc. Natl. Acad. Sci. USA 106, 4331-6 (2009).

23. Xin, K.Q. et al. Immunogenicity and protective efficacy of orally administered recombinant Lactococcus lactis expressing surface-bound HIV Env. Blood 102, 223-228 (2003).

24. Daniel, C. et al. Protection against Yersinia pseudotuberculosis infection conferred by a Lactococcus lactis mucosal delivery vector secreting LcrV. Vaccine 27, 1141-1144 (2009).

25. Robinson, K., Chamberlain, L.M., Schofield, K.M., Wells, J.M. \& Le Page, R.W. Oral vaccination of mice against tetanus with recombinant. Lactococcus lactis. Nat. Biotechnol. 15, 653-657 (2009).

26. Zeng, G. et al. NSOM- and AFM-based nanotechnology elucidates nanostructural and atomic-force features of a $Y$. pestis $V$ immunogencontaining particle vaccine capable of eliciting robust response. Proteomics 9, 1538-1547 (2009).

27. Siegrist, C.A. Mechanisms by which maternal antibodies influence infant vaccine responses: review of hypotheses and definition of main determinants. Vaccine 21, 3406-3412 (2003).
28. Griebel, P.J. Mucosal vaccination of the newborn: an unrealized opportunity. Expert. Rev. Vaccines 8, 1-3 (2009).

29. Conroy, M.E., Shi, H.N. \& Walker, W.A. The long-term health effects of neonatal microbial flora. Curr. Opin. Allergy Clin. Immunol. 9, 197-201 (2009).

30. Latvala, S. et al. Potentially probiotic bacteria induce efficient maturation but differential cytokine production in human monocyte-derived dendritic cells. World J. Gastroenterol. 14, 5570-5583 (2009).

31. Yam, K.K., Pouliot, P., N'diaye, M.M., Fournier, S., Olivier, M. \& Cousineau, B. Innate inflammatory responses to the Gram-positive bacterium Lactococcus lactis. Vaccine 26, 2689-2699 (2008).

32. Niers, L.E. et al. Selection of probiotic bacteria for prevention of allergic diseases: immunomodulation of neonatal dendritic cells. Clin. Exp. Immunol. 149, 344-352 (2007).

33. Debarry, J. et al. Acinetobacter Iwoffii and Lactococcus lactis strains isolated from farm cowsheds possess strong allergy-protective properties. J. Allergy. Clin. Immunol. 119, 1514-1521 (2007).

34. Ramirez, K., Capozzo, A.V., Lloyd, S.A., Sztein, M.B., Nataro, J.P. \& Pasetti, M.F. Mucosally delivered Salmonella typhi expressing the Yersinia pestis $\mathrm{F} 1$ antigen elicits mucosal and systemic immunity early in life and primes the neonatal immune system for a vigorous anamnestic response to parenteral F1 boost. J. Immunol. 182, 1211-1222 (2009).

35. Steen, A. et al. Cell wall attachment of a widely distributed peptidoglycan binding domain is hindered by cell wall constituents. J. Biol. Chem. 278, 23874-23881 (2003)

36. Capozzo, A.V., Cuberos, L., Levine, M.M. \& Pasetti, M.F. Mucosally delivered Salmonella live vector vaccines elicit potent immune responses against a foreign antigen in neonatal mice born to naive and immune mothers. Infect. Immun. 72, 4637-4646 (2004). 